\title{
Study of dark matter physics in non-universal gaugino mass scenario
}

\author{
Junichiro Kawamura ${ }^{a}$ and Yuji Omura ${ }^{b}$ \\ ${ }^{a}$ Department of Physics, Waseda University, \\ Tokyo 169-8555, Japan \\ ${ }^{b}$ Kobayashi-Maskawa Institute for the Origin of Particles and the Universe (KMI), \\ Nagoya University, Nagoya 464-8602, Japan \\ E-mail: junichiro-k@ruri.waseda.jp, yujiomur@kmi.nagoya-u.ac.jp
}

ABSTRACT: We study dark matter physics in the Minimal Supersymmetric Standard Model with non-universal gaugino masses at the unification scale. In this scenario, the specific ratio of wino and gluino masses realizes the electro-weak scale naturally and achieves $125 \mathrm{GeV}$ Higgs boson mass. Then, relatively light higgsino is predicted and the lightest neutral particle, that is dominantly given by the neutral component of higgsino, is a good dark matter candidate. The direct detection of the dark matter is sensitive to not only a higgsino mass but also gaugino masses significantly. The upcoming XENON1T experiment excludes the parameter region where bino or gluino is lighter than about $2.5 \mathrm{TeV}$ if the higgsino and the gaugino mass parameters have same signs. We see that the direct detection of dark matter gives stronger bound than the direct search at the LHC experiment when higgsino gives sizable contribution to the dark matter abundance.

KEYWORDs: Supersymmetry Phenomenology

ArXIV EPRINT: 1703.10379 


\section{Contents}

1 Introduction 1

2 NUGM scenario 2

2.1 Review of NUGM 2

2.2 Mass spectrum of NUGM 4

2.3 LHC bounds 5

3 Dark matter physics $\quad 6$

3.1 Neutralino sector 6

$\begin{array}{ll}3.2 & \text { Thermal relic abundance } \\ 3.3 & \text { Direct }\end{array}$

$\begin{array}{lll}3.3 & \text { Direct detection } & 7\end{array}$

$\begin{array}{lll}3.4 & \text { Indirect detection } & 10\end{array}$

$4 \quad$ Numerical results $\quad 12$

$\begin{array}{llr}5 & \text { Conclusion } & 15\end{array}$

\section{Introduction}

Supersymmetry (SUSY) is a promising candidate for physics beyond the Standard Model (SM). The supersymmetric extension predicts the superpartners of the SM particles, and the masses of the SUSY particles are expected to be at least TeV-scale, in order to explain the origin of the electroweak (EW) scale. ${ }^{1}$ In the Minimal Supersymmetric Standard Model (MSSM), there is a supersymmetric mass parameter, what is called $\mu$-parameter, for higgsino that is the superpartner of Higgs bosons. In order to realize the EW scale without fine-tuning, $\mu$-parameter should be EW-scale. Besides, the lightest particle in the MSSM becomes stable because of R-parity, so that higgsino becomes a good dark matter (DM) candidate if there is no lighter SUSY particle. So far, a lot of efforts are devoted to the SUSY search in the collider experiments and the dark matter observations [3]. There are no decisive signals of the SUSY particles, but higgsino is still one of the possible and attractive DM candidates that reveal the origin of the EW scale.

In the MSSM, there are a lot of parameters, so that we can consider many possibilities of the mass spectrum for the SUSY particles. The direct searches for the SUSY particles as well as the $125 \mathrm{GeV}$ Higgs boson mass measurement at the LHC [4], however, constrain the parameter space strictly. It is getting very difficult to construct SUSY models, as long as the explanation of the EW scale is not discarded. One possible setup to achieve both the $125 \mathrm{GeV}$ Higgs boson mass and the explanation of the EW scale is known as the NonUniversal Gaugino Masses (NUGM) scenario [5, 6]. In this scenario, a suitable ratio of the

\footnotetext{
${ }^{1}$ See for reviews e.g. $[1,2]$.
} 
wino mass to the gluino mass achieves the EW scale and the $125 \mathrm{GeV}$ Higgs boson mass. Then, the $\mu$-parameter is predicted to be close to the EW scale. The current status and the future prospect of the discovery of the SUSY particles at the LHC have been investigated in this scenario [7-9]. We find that the superpartners of top quark and gluon, what are called top squark and gluino, are promising particles to test this scenario. Expected reaches of these SUSY particles decaying to higgsinos are studied in refs. [10-12]. ${ }^{2}$

Note that there are some models that lead such a ratio of the gauginos. One possibility is the mirage mediation [14-16], that is a mixture of the moduli mediation [17-19] and anomaly mediation [20,21]. The phenomenology of the mirage mediation is discussed before the Higgs boson discovery in refs. [22-33] and after that in refs. [34-40]. There are some works to realize the ratio of the gauginos in the GUT models [41] and superstring models [42].

In this kind of SUSY models, higgsino is light because of the explanation of the origin of the EW scale, and the SUSY particle is expected to be discovered in experiments. There are neutral and charged components in higgsino, and the neutral component mixes with bino and wino, and the charged component mixes with wino. ${ }^{3}$ In our scenario, the gauginos are relatively heavy, so that all components of higgsino are light and almost degenerate; in fact, the mass difference is a few $\mathrm{GeV}[7,8]$. Then, higgsino is hard to be detected at the LHC due to the certainly small mass differences. On the other hand, dark matter direct detection experiments can efficiently observe higgsinos, if the neutral component of higgsino slightly mixes with the gauginos and dominates over our universe. It is also interesting that the higgsino mass should be lighter than about $1 \mathrm{TeV}$, if higgsino is thermally produced. Then, our DM mass, that mainly comes from the neutral component of higgsino, is predicted to be between the EW scale and $1 \mathrm{TeV}$.

In this paper, we study dark matter physics in the NUGM scenario. Direct detection experiments are sensitive to not only the higgsino mass itself, but also the gaugino masses, because the higgsino-gaugino mixing gives the most significant contribution to the detection rate. We also discuss the constraints from the LHC experiments, based on the results in refs. [7-9]. We explicitly show the exclusion limit and the future prospect on the plane of the higgsino and the gaugino masses. In the end, we find that this scenario can be fully covered by the future experiments, as far as the gluino mass is below $2.5 \mathrm{TeV}$ in a certain parameter set.

This paper is organized as follows. The NUGM scenario is reviewed in section 2, and we discuss dark matter physics in section 3 . The results of numerical calculations are shown in section 4 . Section 5 is devoted to conclusion.

\section{NUGM scenario}

\section{$2.1 \quad$ Review of NUGM}

The NUGM scenario is known as one of the attractive SUSY models to realize $\mu$-parameter near the EW scale and the $125 \mathrm{GeV}$ Higgs boson mass simultaneously. The $\mu$-parameter

\footnotetext{
${ }^{2}$ There is also a study for the muon g-2 with non-universal gaugino masses [13].

${ }^{3}$ Wino and bino are the superpartners of $\mathrm{SU}(2)_{L}$ and $\mathrm{U}(1)_{Y}$ gauge bosons, respectively.
} 
is related to the EW symmetry breaking scale through the minimization condition for the Higgs potential as

$$
m_{Z}^{2} \simeq 2\left|m_{H_{u}}^{2}\right|-2|\mu|^{2}
$$

where $m_{Z}$ is the Z-boson mass and $m_{H_{u}}^{2}$ is the soft scalar mass squared for the up-type Higgs boson. This relation shows that $|\mu|^{2}$ and $\left|m_{H_{u}}^{2}\right|$ should be around the EW scale to avoid the fine-tuning between those parameters. The $\mu$-parameter is an unique SUSYpreserving parameter in the MSSM. On the other hand, all other dimensional parameters softly break SUSY and would be originated from some mediation mechanisms of SUSY breaking: i.e., the soft SUSY breaking terms would have same origin. Let us assume that the all ratios of soft SUSY breaking parameters are fixed by some mediation mechanisms and the overall scale is given by $M_{0}$. In this assumption, eq. (2.1) corresponds to the relation between $\mu$ and $M_{0}$. In ref. [43], the parameter, $\Delta_{x}$, to measure the sensitivity of the parameter $x$ to the EW scale is introduced:

$$
\Delta_{x}=\left|\frac{\partial \ln m_{Z}^{2}}{\partial \ln x^{2}}\right| \quad\left(x=\mu, M_{0}\right) .
$$

Since $m_{H_{u}}^{2}\left(m_{\mathrm{SUSY}}\right)$ is expressed as a quadratic polynomial function of the boundary conditions, we can derive $\Delta_{\mu}+\Delta_{M_{0}}=1$ at the tree-level and $\Delta_{\mu} \simeq \Delta_{M_{0}}$ is satisfied. Thus the tuning of the $\mu$-parameter represents the degree of tuning to realize the EW symmetry breaking in the model. From the relation eq. (2.1), the tuning measure of the $\mu$-parameter can be written as $\Delta_{\mu}=2|\mu|^{2} / m_{Z}^{2}$ up to radiative corrections to the condition, so that small $|\mu|$ is simply required to avoid the fine-tuning in this assumption. The details of this kind of discussions in the NUGM scenario are shown in refs. [9, 44]. We proceed to study collider and dark matter phenomenology with the NUGM in this assumption. For reference, $\Delta_{\mu}=10,100,250$ correspond to $\mu \sim 200,650,1000 \mathrm{GeV}$, respectively.

In this paper, we assume universal soft scalar mass $m_{0}$ and A-term $A_{0}$, while the gaugino masses $M_{1,2,3}$ are non-universal at the gauge coupling unification scale $\left(\simeq 10^{16} \mathrm{GeV}\right)$. We assume the ratio of two Higgs vacuum expectation values (VEVs) $\tan \beta \equiv\left\langle H_{u}\right\rangle /\left\langle H_{d}\right\rangle=$ 10 throughout this paper. The soft mass squared $m_{H_{u}}^{2}$ at $m_{\mathrm{SUSY}}=1 \mathrm{TeV}$ relates to the boundary conditions at the unification scale as

$$
\begin{aligned}
m_{H_{u}}^{2}\left(m_{\mathrm{SUSY}}\right) \simeq & 0.005 M_{1}^{2}-0.005 M_{1} M_{2}+0.201 M_{2}^{2}-0.021 M_{1} M_{3}-0.135 M_{2} M_{3} \\
& -1.57 M_{3}^{2}+A_{0}\left(0.011 M_{1}+0.065 M_{2}+0.243 M_{3}-0.099 A_{0}\right) \\
& -0.075 m_{0}^{2} .
\end{aligned}
$$

This relation shows that the contribution from the gluino mass is dominant among the renormalization group (RG) effects, but we find that the gluino mass contribution can be canceled by the RG effects from the other gaugino masses $M_{1,2}$. In particular, the $M_{2}^{2}$ term cancels the $M_{3}^{2}$ term if the ratio of $M_{2} / M_{3}$ satisfies $M_{2} / M_{3} \simeq 3$-4. Similarly, the top squark mass parameters $m_{\tilde{t}_{L}}^{2}, m_{\tilde{t}_{R}}^{2}$ and $A_{t}$ at $m_{\mathrm{SUSY}}=1 \mathrm{TeV}$ are related to the boundary 
conditions as

$$
\begin{aligned}
m_{\tilde{t}_{L}}^{2}\left(m_{\mathrm{SUSY}}\right) \simeq & -0.007 M_{1}^{2}-0.002 M_{1} M_{2}+0.354 M_{2}^{2}-0.007 M_{1} M_{3}-0.051 M_{2} M_{3} \\
& +3.25 M_{3}^{2}+\left(0.004 M_{1}+0.025 M_{2}+0.094 M_{3}-0.039 A_{0}\right) A_{0} \\
& +0.622 m_{0}^{2} \\
m_{\tilde{t}_{R}}^{2}\left(m_{\mathrm{SUSY}}\right) \simeq & 0.044 M_{1}^{2}-0.003 M_{1} M_{2}-0.158 M_{2}^{2}-0.014 M_{1} M_{3}-0.090 M_{2} M_{3} \\
& +2.76 M_{3}^{2}+\left(0.008 M_{1}+0.044 M_{2}+0.162 M_{3}-0.066 A_{0}\right) A_{0} \\
& +0.283 m_{0}^{2} \\
A_{t}\left(m_{\text {SUSY }}\right) \simeq & -0.032 M_{1}-0.237 M_{2}-1.42 M_{3}+0.277 A_{0}
\end{aligned}
$$

We see that $A_{t}\left(m_{\mathrm{SUSY}}\right)$ increases and $m_{\tilde{t}_{R}}^{2}\left(m_{\mathrm{SUSY}}\right)$ decreases as the wino mass $M_{2}$ increases. Note that the latter effect is induced by the top Yukawa coupling. As a result, the ratio $A_{t}^{2} / \sqrt{m_{\tilde{t}_{L}}^{2} m_{\tilde{t}_{R}}^{2}}$ increases and the SM-like Higgs boson mass around $125 \mathrm{GeV}$ can be achieved due to the relatively large wino.

\subsection{Mass spectrum of NUGM}

We see that the suitable wino-to-gluino mass ratio reduces the $\mu$-parameter and also enhances the Higgs boson mass. Besides, some of sparticle masses are within reaches of the LHC experiment thanks to the sizable left-right mixing of the top squarks $[7,8]$.

When the wino mass is large, left-handed sparticles become heavy due to the RG evolution. The right-handed slepton masses are determined by the bino mass, while the right-handed squark masses mainly depend on both the gluino and bino masses. The bino mass plays a crucial role in shifting the top squark mass, as well. This means that the bino mass have to be so heavy that the top squark mass is enough heavy to be consistent with the LHC results.

Another important point derived from the relatively heavy bino and wino is that the mass differences among the components of higgsino become small. The mass differences are induced by the mixing with higgsino and gauginos, so that these are suppressed by the bino and wino masses as explicitly shown in next section. The mass differences among the components of higgsino are typically $2 \mathrm{GeV}$ as shown in ref. [7]. This small mass difference makes it difficult to detect higgsino directly at the LHC, because their daughter particles are too soft to be distinguished from backgrounds and their lifetimes are too short to be recognized as charged tracks unlike the case that wino is the lightest SUSY particle (LSP) $[45] .{ }^{4}$ This feature also indicates that we can treat all of the particles from higgsino as invisible particles at the LHC.

Let us summarize the important features of our mass spectrum discussed below:

- All gauginos are $\mathcal{O}(1) \mathrm{TeV}$.

- The higgsino mass is between the EW scale and $1 \mathrm{TeV}$, and the mass differences are $\mathcal{O}(1) \mathrm{GeV}$.

- Right-handed top squark is relatively light.

\footnotetext{
${ }^{4}$ There are recent works to study searching for charged higgsinos that exploit their relatively long lifetime $[46,47]$.
} 


\subsection{LHC bounds}

In our scenario, the top squark and the gluino are the good candidates to be detected at the LHC. The current exclusion limit and the future prospect have been studied in refs. [7-9].

In the NUGM scenario, a top squark decays as $\tilde{t}_{1} \rightarrow t \tilde{\chi}_{1,2}^{0} / b \tilde{\chi}_{1}^{ \pm}$where each branching fraction is $50 \%$ as long as the mass difference between the top squark and each of the higgsino-like particles is significantly larger than the top quark mass. Note that the neutralinos consist of higgsino that slightly mixes with wino and bino in our scenario. The relevant top squark searches at the LHC are discussed in ref. [48] and ref. [49]. The former analysis aims to a pair of bottom squarks that decay as $\tilde{b}_{1} \tilde{b}_{1} \rightarrow b \tilde{\chi}^{0} b \tilde{\chi}^{0}$. This gives same signal as $\tilde{t}_{1} \tilde{t}_{1} \rightarrow b \tilde{\chi}^{ \pm} b \tilde{\chi}^{ \pm}$in the NUGM scenario. The latter analysis aims to hadronically decaying top squarks, $\tilde{t}_{1} \tilde{t}_{1} \rightarrow t \tilde{\chi}^{0} t \tilde{\chi}^{0} \rightarrow b j j \tilde{\chi}^{0} b j j \tilde{\chi}^{0}$. In ref. [49], the signal regions require more than 4 jets, where 2 of these should be b-tagged. Such signal regions will be sensitive to events $\tilde{t}_{1} \tilde{t}_{1} \rightarrow t(\rightarrow b j j) \tilde{\chi}^{0} b \tilde{\chi}^{ \pm}$in the NUGM scenario, although this analysis is not completely optimized. This decay pattern is realized in almost half of the events with the pair produced top squarks if the mass difference between the top squark and higgsino is enough large. Thus this channel that targets to the hadronically decaying top squark is sensitive to the large mass difference region, while the former channel that targets to bottom squarks decaying to a bottom quark and a neutralino is sensitive to the mass degenerate region. Referring the analysis in ref. [9], top squark lighter than $800 \mathrm{GeV}$ is excluded if $\mu \lesssim 200 \mathrm{GeV}$ is satisfied, and top squark lighter than $600 \mathrm{GeV}$ is excluded in the range with $200 \mathrm{GeV} \lesssim \mu \lesssim 270 \mathrm{GeV}$. There is no exclusion limit for top squarks if $\mu$ is greater than $270 \mathrm{GeV}$.

In present scenario, a gluino decays as $\tilde{g} \rightarrow t \tilde{t}_{1} \rightarrow t+t \tilde{\chi}^{0} / b \tilde{\chi}^{ \pm}$. Hence, the signal from the gluino pair production is expected to have 4 b-tagged jets, jets/leptons coming from 2-4 W-bosons and large missing energies in the final state. The analysis in ref. [50] aims to this type of signals, and we refer the exclusion limit obtained in ref. [9]. Gluino lighter than $1.8 \mathrm{TeV}$ is excluded if the $\mu$-parameter is less than $800 \mathrm{GeV}$. The bound is relaxed if the mass difference is smaller than about $300 \mathrm{GeV}$.

Note that there is another channel, $\tilde{g} \rightarrow g \tilde{\chi}^{0}$, that is induced by the top squark loop. If the mass difference between gluino and higgsino is near or less than the top quark mass, this decay channel becomes important. We need to consider the limits based on data such as ref. [51], but it is beyond the scope of this paper.

Let us comment on the case with light bino. If gluino is enough heavy, bino can be as light as higgsino and top squark can also decay to bino. The decay is, however, usually suppressed unless bino is significantly lighter than higgsino because the coupling of bino with top squark is much weaker than the one of higgsinos because of the top Yukawa coupling. Such a light bino is less attractive from the experimental point of view. If the bino mass is light, gluino has to be much heavier than the experimental reach in order to shift the top squark mass. Then, the light bino case would be unfavorable from the naturalness point of view. Furthermore, it is known that bino LSP tends to overclose the universe and some dilution mechanisms are necessary. 


\section{Dark matter physics}

\subsection{Neutralino sector}

In our study, we assume that the signs of all the gaugino masses are positive and the sign of the $\mu$-parameter is either negative or positive. After the EW symmetry breaking, gauginos and higgsino are mixed each other. The neutralino mass matrix in a basis of $\psi=\left(\tilde{B}, \tilde{W}, \tilde{H}_{d}^{0}, \tilde{H}_{u}^{0}\right)$ is given by

$$
M_{\tilde{\chi}}=\left(\begin{array}{cccc}
M_{1} & 0 & -c_{\beta} s_{W} m_{Z} & s_{\beta} s_{W} m_{Z} \\
0 & M_{2} & c_{\beta} c_{W} m_{Z} & -s_{\beta} c_{W} m_{Z} \\
-c_{\beta} s_{W} m_{Z} & c_{\beta} c_{W} m_{Z} & 0 & -\mu \\
s_{\beta} s_{W} m_{Z} & -s_{\beta} c_{W} m_{Z} & -\mu & 0
\end{array}\right),
$$

where $c_{\beta}=\cos \beta, s_{\beta}=\sin \beta, c_{W}=\sin \theta_{W}$ and $s_{W}=\sin \theta_{W}$ are defined and $\theta_{W}$ is the Weinberg angle. This matrix is diagonalized by an unitary matrix $N$ as

$$
\psi_{i}=N_{i j} \tilde{\chi}_{j} \text { and } N^{\dagger} M_{\tilde{\chi}} N=\operatorname{diag}\left(m_{\tilde{\chi}_{1}}, m_{\tilde{\chi}_{2}}, m_{\tilde{\chi}_{3}}, m_{\tilde{\chi}_{4}}\right) \text {. }
$$

The masses, $m_{\tilde{\chi}_{1}}, m_{\tilde{\chi}_{2}}, m_{\tilde{\chi}_{3}}$ and $m_{\tilde{\chi}_{4}}$ approach to $M_{1}, M_{2}, \mu$, and $-\mu$ in the limit that $m_{Z}$ is vanishing, respectively. The mass eigenstate $\tilde{\chi}_{3}\left(\tilde{\chi}_{4}\right)$ becomes the lightest one if the $\mu$-parameter is positive (negative) and $|\mu|<M_{1}, M_{2}$.

The neutralino-neutralino-Higgs coupling, $\mathcal{L} \ni(1 / 2) \lambda_{h n n} h \overline{\tilde{\chi}}_{n} \tilde{\chi}_{n}$, is given by

$$
\lambda_{h n n}=g\left(s_{\alpha} N_{3 n}+c_{\alpha} N_{4 n}\right)\left(N_{2 n}-t_{W} N_{1 n}\right),
$$

where $t_{W}, s_{\alpha}$ and $c_{\alpha}$ are short for $\tan \theta_{W}, \sin \alpha$ and $\cos \alpha$, respectively. $\alpha$ is a mixing angle of the Higgs boson. The mixing matrix is given by

$$
\begin{aligned}
& \left(N_{11}, N_{21}, N_{31}, N_{41}\right)=\left(1,0,-\frac{m_{Z} s_{W}\left(c_{\beta} M_{1}+s_{\beta} \mu\right)}{M_{1}^{2}-\mu^{2}}, \frac{m_{Z} s_{W}\left(c_{\beta} \mu+s_{\beta} M_{1}\right)}{M_{1}^{2}-\mu^{2}}\right), \\
& \left(N_{12}, N_{22}, N_{32}, N_{42}\right)=\left(0,1, \frac{m_{Z} c_{W}\left(c_{\beta} M_{2}+s_{\beta} \mu\right)}{M_{2}^{2}-\mu^{2}},-\frac{m_{Z} c_{W}\left(c_{\beta} \mu+s_{\beta} M_{2}\right)}{M_{2}^{2}-\mu^{2}}\right), \\
& \left(N_{13}, N_{23}, N_{33}, N_{43}\right)=\frac{1}{\sqrt{2}}\left(\frac{m_{Z} s_{W}\left(c_{\beta}+s_{\beta}\right)}{M_{1}-\mu},-\frac{m_{Z} c_{W}\left(c_{\beta}+s_{\beta}\right)}{M_{2}-\mu}, 1,-1\right), \\
& \left(N_{14}, N_{24}, N_{34}, N_{44}\right)=\frac{1}{\sqrt{2}}\left(\frac{m_{Z} s_{W}\left(c_{\beta}-s_{\beta}\right)}{M_{1}+\mu},-\frac{m_{Z} c_{W}\left(c_{\beta}-s_{\beta}\right)}{M_{2}+\mu}, 1,1\right),
\end{aligned}
$$

where $m_{Z} \ll\left|M_{1,2} \pm \mu\right|$ is assumed.

\subsection{Thermal relic abundance}

It is known that the thermal relic density of the purely higgsino LSP saturates the universe when the higgsino mass is about $1 \mathrm{TeV}[52,53]$. If we assume that there is no dilution effect after the thermal production of the LSP, the higgsino-like LSP heavier than $1 \mathrm{TeV}$ overcloses the universe and is cosmologically excluded unless the higgsino and another sparticle, such as a top squark, are so degenerate that co-annihilation processes between them reduce the relic density. 
Let us comment on possibilities that gauginos contribute to dark matter considerably. In our scenario, the wino mass should be as large as the gluino mass at the $\mathrm{TeV}$ scale and it hardly contributes to the dark matter. The bino mass can be as light as the higgsino mass if the gluino mass is enough large to keep the top squark mass. It was interesting that the welltempered bino-higgsino LSP explains the observed abundance in the thermal scenario [54], but most of parameter space has been already excluded by the direct detections as will be discussed later. ${ }^{5}$

In our scenario, the relic DM abundance thermally produced may not be sufficient to satisfy the observed DM abundance in our universe. When we denote the relic abundance of the LSP as $\Omega_{\chi} h^{2}$, we can simply consider two possibilities to saturate the observed value, $\Omega_{\mathrm{obs}} h^{2}=0.1188 \pm 0.0001[56]$ :

(A) $\Omega_{\chi} h^{2}$ is given by the thermal production and $\Omega_{\chi} h^{2}=\Omega_{\mathrm{th}} h^{2} \leq \Omega_{\mathrm{obs}} h^{2}$ is satisfied where $\Omega_{\mathrm{th}}$ is the thermal relic density of the LSP. Detection rates for the LSP at dark matter detections are suppressed by a fraction $\xi \equiv \Omega_{\chi} / \Omega_{\mathrm{obs}}=\Omega_{\mathrm{th}} / \Omega_{\mathrm{obs}} \equiv \xi_{\mathrm{th}}$.

(B) $\Omega_{\chi} h^{2}=\Omega_{\mathrm{obs}} h^{2}$ is always satisfied, assuming non-thermal production of LSP works. Detection rates for the LSP are simply determined by cross sections for relevant processes and the suppression factor is unity: $\xi \equiv \Omega_{\chi} / \Omega_{\mathrm{obs}}=1$.

In the case (A), the LSP may not saturate our universe, depending on the parameter region. Then, we need other DM candidates such as axion to achieve the observed relic abundance of the DM. We also assume that the thermal relic density of the LSP is not changed by introducing other DM candidates to the MSSM. The direct detection rate is suppressed by $\xi_{\text {th }}$ and the indirect detection rate is suppressed by $\xi_{\text {th }}^{2}$. The difference of the scalings comes from the fact that the relevant process is scattering of the LSP against nucleons in the direct detection, while it is coannihilation of two LSPs into SM particles in the indirect detection.

In the case (B), we simply assume that the LSP dominates our universe and satisfies $\xi=1$. We do not explicitly calculate the relic abundance, but several mechanisms for the non-thermal productions have been proposed so far. For instance, it is known that the decays of long-lived heavy particles, such as gravitino, saxion and moduli field, can significantly produce the LSP after the LSP is frozen out from the thermal bath [57-60].

Throughout this paper, we focus on the region where $\xi_{\text {th }} \leq 1$ corresponding to $\mu \lesssim$ 1.0 TeV. We note that region with $\xi_{\text {th }} \geq 1$ is not truly excluded in the case (B), but such region is less attractive because the degree of tuning of the $\mu$-parameter to realize the $\mathrm{EW}$ scale is severer than $\Delta_{\mu} \gtrsim 250$ corresponding to $0.4 \%$ tuning.

\subsection{Direct detection}

The direct detection for dark matter is a promising way to probe the neutralino sector of the MSSM. The current limits on the spin-independent and spin-dependent cross sections

\footnotetext{
${ }^{5}$ There are narrow regions where the thermal bino-higgsino LSP explains the abundance by the Higgsor Z-boson resonances without tension with the DM direct detection experiments [55].
} 
are given by the XENON100 [61-63], LUX [64, 65], PANDAX-II [66, 67] and PICO [68, 69]. The XENON1T [70] and LZ [71] will cover wider range in near future.

Let us discuss spin-independent cross section of neutralino scattering with nucleons. Note that the limits on the gaugino masses from the spin-independent cross section are stronger than those from the spin-dependent cross section in most cases.

At tree-level, spin-independent scatterings are induced by the t-channel Higgs boson exchange and the s-channel squark exchange. Since only one top squark is light in the NUGM scenario, the latter contribution is negligibly small. The mixing between gauginos and higgsino are important in the Higgs boson exchange, because the LSP-LSP-Higgs coupling in the mass eigenstate basis is originated from the gaugino-higgsino-Higgs couplings in the gauge eigenstate basis. In the limit of $m_{Z} \ll\left|M_{1,2} \pm \mu\right|$, the mixing effects are suppressed by $m_{Z} /\left|M_{1,2} \pm \mu\right|$ as shown in eqs. (3.6) and (3.7).

It has been shown that there is a parameter set to lead vanishing gaugino-higgsino mixing, what is called the blind spot [72]. As we see eqs. (3.3), (3.4) and (3.5), the mixing is proportional to $M_{1,2}+\mu \sin 2 \beta$, so that the mixing vanishes when the relative signs of $M_{1,2}$ and $\mu$ are opposite, and $\left|M_{1,2}\right| \lesssim|\mu|$ and $\tan \beta \gtrsim 1$ are satisfied. Thus the blind spot appears only in the gaugino-like LSP scenario.

Note that the mixing is suppressed when the LSP is higgsino-like and signs of $\mu$ and $M_{1,2}$ are opposite, as we can see from eqs. (3.6) and (3.7). Since the mixing is proportional to $1 \pm \sin 2 \beta$, smaller $\tan \beta$ induces larger enhancement (suppression) for the same (opposite) sign. We need $\tan \beta \gtrsim 10$ in order to realize the SM-like Higgs boson mass unless the sparticle masses are much heavier than $1 \mathrm{TeV}$, so that such effect is at most 20\%-level. Thus we conclude that the gaugino-higgsino mixing is sizable and the factor, $1 \pm \sin 2 \beta$, leads significant difference between the positive and the negative $\mu$-parameter cases in the DM scattering cross section.

The spin-independent cross section per nucleon at the tree-level can be written as

$$
\sigma_{N}^{\mathrm{SI}}=\frac{g^{2}}{4 \pi} \frac{m_{N}^{4}}{m_{h}^{4} m_{W}^{2}}\left(1+\frac{m_{N}}{m_{\chi}}\right)^{-2}\left[\frac{2}{9}+\frac{7}{9} \sum_{q=u, d, s} f_{T_{q}}^{N}\right]^{2} \lambda_{h \chi \chi}^{2},
$$

where $m_{N}$ is the nucleon mass and $m_{N} f_{T_{q}}^{N}=\left\langle N\left|m_{q} \bar{q} q\right| N\right\rangle$. In the decoupling limit $m_{A} \gg$ $m_{Z}$ that is a good approximation for our case, using eqs. (3.6) and (3.7), the LSP-LSPHiggs coupling $\lambda_{h \chi \chi}$ is derived from eq. (3.3):

$$
\lambda_{h \chi \chi}=\frac{g}{2}\left(1 \pm s_{2 \beta}\right) c_{W}\left(\frac{m_{Z}}{M_{2}-|\mu|}+t_{W}^{2} \frac{m_{Z}}{M_{1}-|\mu|}\right)
$$

where \pm corresponds to a sign of the $\mu$-parameter.

We list the explicit values of masses and observables at the sample points in table 1 . In our numerical analysis, We use softsusy-3.5.1 [88] to calculate the RG effects and the mass spectrum of sparticles and Higgs bosons. Their width and branching ratios are calculated by SDECAY and HDECAY [89-91]. Note that the dark matter observables are calculated by micrOmega-4.2.5 [73-75]. We can see that the A-term is same order as other input 


\begin{tabular}{|c|c|c|c|c|}
\hline input $[\mathrm{GeV}]$ & (a) & (b) & (c) & (d) \\
\hline$\mu$ & -250 & 250 & -1000 & 1000 \\
\hline$M_{1}\left(M_{U}\right)$ & 10000 & 10000 & 5000 & 5000 \\
\hline$M_{3}\left(M_{U}\right)$ & 1000 & 1000 & 1500 & 1500 \\
\hline$m_{0}\left(M_{U}\right)$ & 1000 & 1000 & 1000 & 1000 \\
\hline \multicolumn{5}{|l|}{ output $[\mathrm{GeV}]$} \\
\hline$M_{2}\left(M_{U}\right)$ & 4223 & 4175 & 4698 & 4504 \\
\hline$A_{0}\left(M_{U}\right)$ & -2378 & -2325 & -1916 & -1657 \\
\hline \multicolumn{5}{|l|}{ mass $[\mathrm{GeV}]$} \\
\hline$m_{h}$ & 125.0 & 125.0 & 125.0 & 125.0 \\
\hline$m_{A}$ & 3349 & 3326 & 3351 & 3248 \\
\hline$m_{\tilde{t}_{1}}$ & 1606 & 1636 & 1431 & 1581 \\
\hline$m_{\tilde{t}_{2}}$ & 2780 & 2762 & 3582 & 3520 \\
\hline$m_{\tilde{g}}$ & 2250 & 2250 & 3225 & 3223 \\
\hline$m_{\tilde{\chi}_{1}^{0}}$ & 258.8 & 255.7 & 1016 & 1013 \\
\hline$m_{\tilde{\chi}_{2}^{0}}$ & 260.5 & 258.3 & 1019 & 1017 \\
\hline$m_{\tilde{\chi}_{3}^{0}}$ & 3438 & 3400 & 2239 & 2237 \\
\hline$m_{\tilde{\chi}_{4}^{0}}$ & 4455 & 4454 & 3839 & 3682 \\
\hline$m_{\tilde{\chi}_{1}^{ \pm}}$ & 260.5 & 257.1 & 1018 & 1015 \\
\hline$m_{\tilde{\chi}_{2}^{ \pm}}$ & 3439 & 3400 & 3840 & 3682 \\
\hline \multicolumn{5}{|l|}{ observables } \\
\hline$\Omega_{\chi} h^{2}$ & $7.82 \times 10^{-3}$ & $7.58 \times 10^{-3}$ & $1.14 \times 10^{-1}$ & $1.16 \times 10^{-1}$ \\
\hline$\langle\sigma v\rangle_{0} \times 10^{25}\left[\mathrm{~cm}^{3} / \mathrm{s}\right]$ & 1.39 & 1.42 & 0.104 & 0.105 \\
\hline $\operatorname{Br}\left(\chi \chi \rightarrow W^{+} W^{-}\right)$ & 0.533 & 0.535 & 0.488 & 0.489 \\
\hline $\operatorname{Br}(\chi \chi \rightarrow Z Z)$ & 0.436 & 0.435 & 0.408 & 0.407 \\
\hline$\sigma_{\mathrm{SD}} \times 10^{-6}[\mathrm{pb}]$ & 1.096 & 1.138 & 0.1677 & 0.1757 \\
\hline$\sigma_{\mathrm{SI}} \times 10^{-11}[\mathrm{pb}]$ & 3.499 & 8.505 & 8.918 & 22.37 \\
\hline$\sigma_{\mathrm{SI}}^{h} \times 10^{-11}[\mathrm{pb}]$ & 3.302 & 7.793 & 7.853 & 19.50 \\
\hline
\end{tabular}

Table 1. Values of boundary conditions at the unification scale $M_{U}$, Higgs boson masses, sparticle masses and dark matter observables at several sample points.

parameters, but the Higgs boson mass is about $125 \mathrm{GeV}$ owing to the suitable wino-togluino mass ratio. The top squark mass is about $1.5 \mathrm{TeV}$ and the gluino mass is $2-3 \mathrm{TeV}$, so that they could be in the reach of the HL-LHC. The bino and wino masses are between $2 \mathrm{TeV}$ and $5 \mathrm{TeV}$ and they are far beyond the experimental reach of the LHC experiment.

From the naturalness point of view, we are especially interested in the low-scale $\mu$ scenarios. When $|\mu|=250(1000) \mathrm{GeV}$ in the samples (a), (b), (c) and (d), the thermal relic abundance is $\sim 0.01(0.1)$. The self-annihilation rate of the neutralinos in the zerovelocity limit, denoted by $\langle\sigma v\rangle_{0}$, is $\mathcal{O}(0.1-1.0) \times 10^{-25}\left[\mathrm{~cm}^{3} / \mathrm{s}\right]$ and they are dominantly annihilating in pairs into weak gauge bosons. These processes are induced by the t-channel neutralino or chargino exchange, and then the rate is determined by the higgsino mass itself. These are important for the indirect detections as discussed below. 
We also show the spin-dependent and spin-independent LSP-proton cross sections, $\sigma_{\mathrm{SD}}, \sigma_{\mathrm{SI}}$, calculated by using micrOMEGA-4.2.5 [73-75]. $\sigma_{\mathrm{SI}}^{h}$ is obtained from eqs. (3.8) and (3.9), where $f_{T_{q}}^{p}$ are taken same as the values adopted in micrOMEGA [76]. We can see the SI cross section is well described by the tree-level Higgs-exchanging process, but there are small deviations from the results of micrOMEGA.

A dominant source for the deviation come from the QCD corrections to the heavy quark matrix elements [77], which enhance the cross section about $10 \%$ against the tree-level contribution. Besides, the top squarks could give contribution to the cross section, when a mass difference $m_{\tilde{t}_{1}}^{2}-m_{\tilde{\chi}}^{2}$ is small. However, it is known that the leading contribution, which is suppressed by $\left(m_{\tilde{t}_{1}}^{2}-m_{\tilde{\chi}}^{2}\right) m_{t}^{2}$, is proportional to the size of non-trivial mixing of the top squarks [78]. The top squark is almost right-handed in our scenario and thus such contribution can not be sizable. We take the top squark corrections derived in ref. [78] into account, and confirm that these are about $1 \%$ against the tree-level countribution at the sample (d) and fewer for the other sample points. We have checked that our results agree with the results of micrOMEGA exhibited in table 1 within several \%-level after including these effects. There are potentially sizable corrections from neutralino/Z-boson and chargino/W-boson mediated loop diagrams, where the neutralino and chargino are higgsino-like, but these are almost canceled out among them as shown in ref. [79].

\subsection{Indirect detection}

Let us comment on indirect detections for the dark matter. A pair of neutralinos decay to $W^{+} W^{-}$or $Z Z$ with the zero-velocity cross section: that is $\mathcal{O}\left(10^{-25}\right)\left[\mathrm{cm}^{3} / \mathrm{s}\right]$ as shown in table 1 .

One of the most promising observables may be the neutrino flux from the sun. The capture rate of neutralino by the sun is determined by the interaction between neutralino and nucleons. Since the spin-dependent cross section is much larger than the spin-independent one, the observations would give significant bounds on the spin-dependent cross section. The weak bosons produced by the annihilation of dark matter decay to neutrinos. The observed limit of neutrinos given by the IceCube is $3.76 \times 10^{-5} \mathrm{pb}$ when the dark matter mass is $500 \mathrm{GeV}$ and they decay to $\mathrm{W}$-bosons exclusively [80]. This limit is comparable to the expected limit at the XENON1T [61]. We will see that exclusion limits for the parameter space from the XENON1T are much weaker than limits from the spin-independent cross section, so that the current limit from IceCube experiment can not be important one.

Cosmic ray observations such as photons, positrons and anti-protons could be powerful tools to detect dark matter. These limits of the annihilation cross section of DM reach to $\mathcal{O}\left(10^{-25}\right)\left[\mathrm{cm}^{3} / \mathrm{s}\right]$ and the parameter region discussed in present paper is competing with these bounds. We consider the recent experimental results obtained by the FermiLAT [81] and AMS-02 [82]. The former observes gamma rays coming from the dwarf spheroidal satellite galaxies (dSphs) of the Milky Way and the latter observes anti-protons coming from dark matter annihilations in the Milky Way. We refer the exclusion limit from the AMS-02 experiment obtained in the analysis [83]. ${ }^{6}$ The Fermi-LAT experiment also

\footnotetext{
${ }^{6}$ Similar analysis is done in ref. [84].
} 


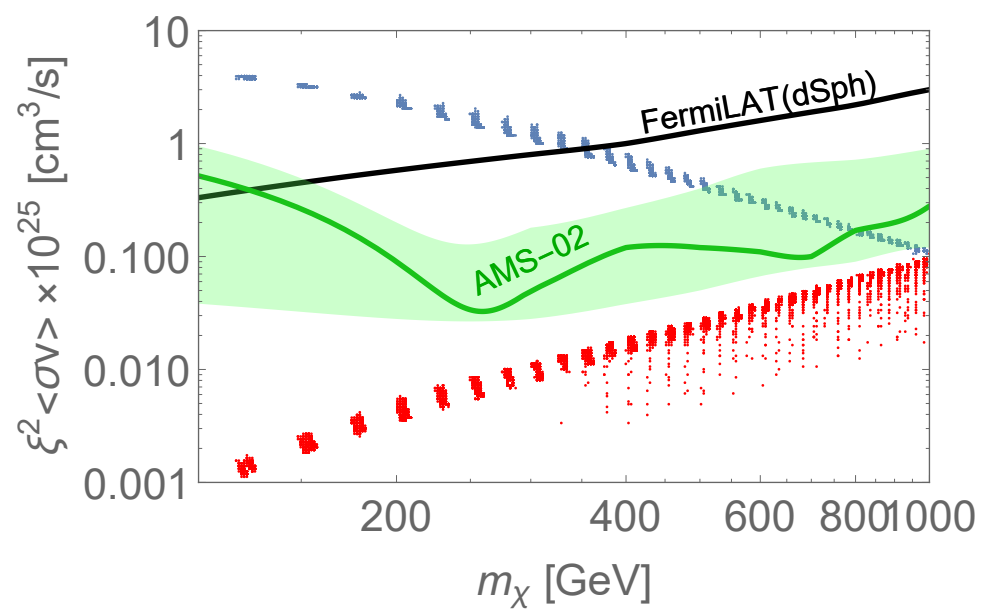

Figure 1. Exclusion limits and expected values in the NUGM scenario of the dark matter annihilation cross section. The blue (red) dots correspond to $\xi=1\left(\xi=\xi_{\text {th }}\right)$.

observes gamma-rays coming from the galactic center and this potentially gives significant constraints on the dark matter annihilation rate. However, the results are highly dependent on dark matter density profiles [85], so that we do not discuss about this in present paper.

Figure 1 shows the upper limits on the annihilation cross section from the recent results of the Fermi-LAT (black line) and the AMS-02 (green line). The uncertainty of the limit from the AMS-02 is shown by the green band, because the limit from the AMS-02 experiment still has large uncertainty. The dots are predictions from the NUGM scenario and obtained by the parameter scanning to draw figures in next section. We plot the points with $M_{1} \geq 2.5 \mathrm{TeV}$ at the unification scale. The blue dots indicate the lightest neutralino mass and the annihilation rate itself and are predicted in the case (B) where $\xi=1$, but it is multiplied by $\xi_{\text {th }}^{2} \equiv\left(\Omega_{\mathrm{th}} / \Omega_{\mathrm{obs}}\right)^{2}$ for the red dots corresponding to the case (A). Since the higgsino-like dark matter dominantly annihilate to W-bosons or Z-bosons by the t-channel exchange of the higgsino-like chargino or neutralino, the annihilation rate is mostly determined by the higgsino mass itself and almost independent of other parameters. We see that the Fermi-LAT result excludes the neutralino lighter than about $300 \mathrm{GeV}$ and the AMS-02 excludes the neutralino lighter than about $800_{+200}^{-300} \mathrm{GeV}$ if the LSP saturates the dark matter and $\xi=1$. On the other hand, the indirect detections do not give limits if the annihilation rate is suppressed by the factor $\xi_{\mathrm{th}}^{2}$. Exclusion limits on the higgsino dark matter produced from some non-thermal processes at the Fermi-LAT and the future planned CTA experiments [86] have been discussed in ref. [87].

In the case (B), where $\xi=1$, the higgsino lighter than about $500 \mathrm{GeV}$ has been excluded even in the loosest case within the uncertainty. This means that the degree of tuning of the $\mu$-parameter is worse than a few \%-level. Note that this limit is independent of other parameters as long as the higgsino is the dominant component of the LSP. For this reason, we will not draw the limits from the indirect detections on figures in the next section. The conservative limit for the higgsino mass is about $500 \mathrm{GeV}$ if $\xi=1$, while there is no bound for the higgsino mass if $\xi=\xi_{\text {th }}$. 


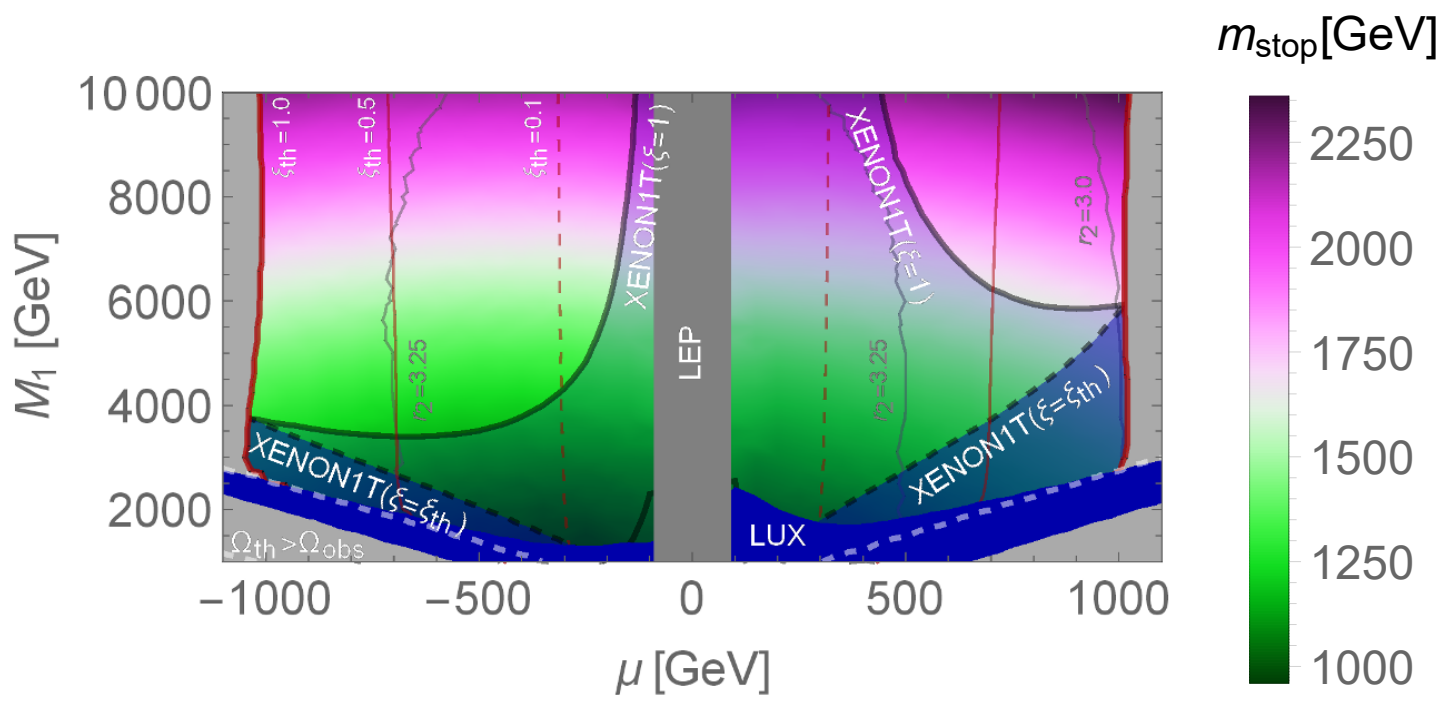

Figure 2. Values of the dark matter observables with $M_{3}=1.5 \mathrm{TeV} . \xi_{\mathrm{th}} \equiv \Omega_{\mathrm{th}} / \Omega_{\mathrm{obs}}=1$ is achieved in the red bands around $|\mu|=1 \mathrm{TeV}$, and $\xi_{\text {th }}=0.5(0.1)$ on the solid (dashed) red lines. $\Omega_{\mathrm{th}} h^{2}>\Omega_{\mathrm{obs}} h^{2}$ in the light gray region. The LUX experiment excludes the blue bands if $\xi=1$ and region below the white dashed lines if $\xi=\xi_{\text {th }}$. The region below the dark solid (dashed) blue lines where is the transparent blue region will be covered by the XENON1T experiment if $\xi=1\left(\xi_{\text {th }}\right)$. The gray lines show the wino-to-gluino mass ratio $r_{2} \equiv M_{2} / M_{3}$ at the unification scale. The gray region where $|\mu|<90 \mathrm{GeV}$ is excluded by the LEP experiment.

\section{Numerical results}

Based on the above discussion, we summarize the experimental bounds and show the allowed region.

Figure 2 shows the allowed region for the dark matter observables, the top squark mass and exclusion limits from the collider experiments. We assume $m_{0}=1 \mathrm{TeV}, M_{3}=1.5 \mathrm{TeV}$ at the unification scale and $A_{0}, M_{2}$ are chosen to realize the SM-like Higgs boson mass and the $\mu$-parameter at each point. We take the ratio of the Higgs VEVs as $\tan \beta=10$.

The red lines represent the thermal relic density of the neutralino, where the solid (dashed) lines correspond to $\xi_{\text {th }} \equiv \Omega_{\text {th }} / \Omega_{\text {obs }}=0.5(0.1) . \quad \Omega_{\chi} h^{2}=\Omega_{\mathrm{obs}} h^{2}=0.1188 \pm$ 0.0001 [56] is achieved in the red bands around $|\mu| \simeq 1 \mathrm{TeV}$. The thermal relic density of the dark matter exceeds the observed value, $\Omega_{\chi} h^{2}>\Omega_{\mathrm{obs}} h^{2}$, in the light gray region. If we allow only $\mathcal{O}(10) \%$ fine-tuning for the EW scale, $|\mu|$ should be $\mathcal{O}(100) \mathrm{GeV}$, as discussed in section 2.1. In such region, $\xi_{\text {th }}=\mathcal{O}(0.1)$ in the case $(\mathrm{A})$ and some mechanisms to compensate the DM relic density are required.

Note that the gray region at $|\mu| \leq 90 \mathrm{GeV}$ is excluded by the LEP experiment [92]. Although the charged and neutral components of higgsino are certainly degenerate, they can be probed by the mono-photon channel. The background color represent the mass of the lightest top squark. The purple line around $M_{1} \lesssim 2.0 \mathrm{TeV}$ and $\mu \simeq-100 \mathrm{GeV}$ is the expected exclusion limits for the spin-dependent cross section from the XENON1T experiment [61]. 


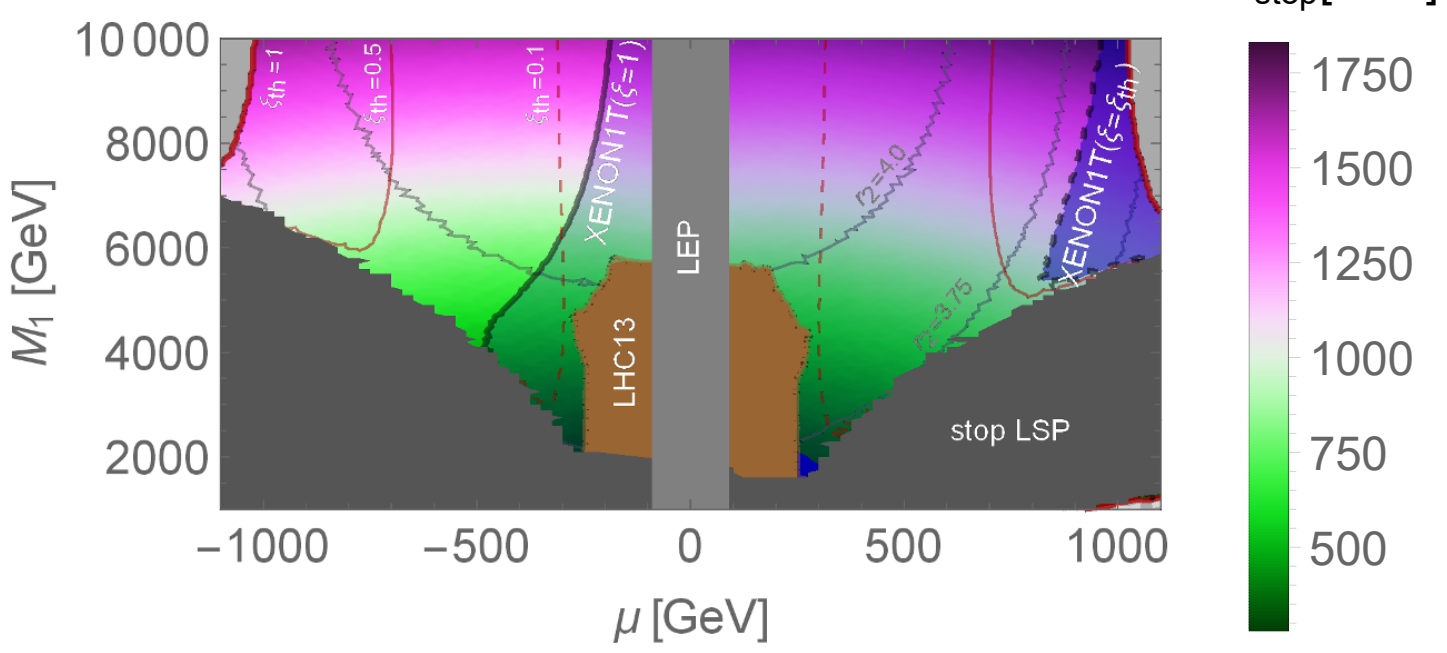

Figure 3. Values of the dark matter observables with $M_{3}=1.0 \mathrm{TeV}$. Meanings of the lines and regions are same as in figure 2. In addition, the brown region is excluded by the top squark search at the LHC and the top squark becomes LSP in the dark gray region.
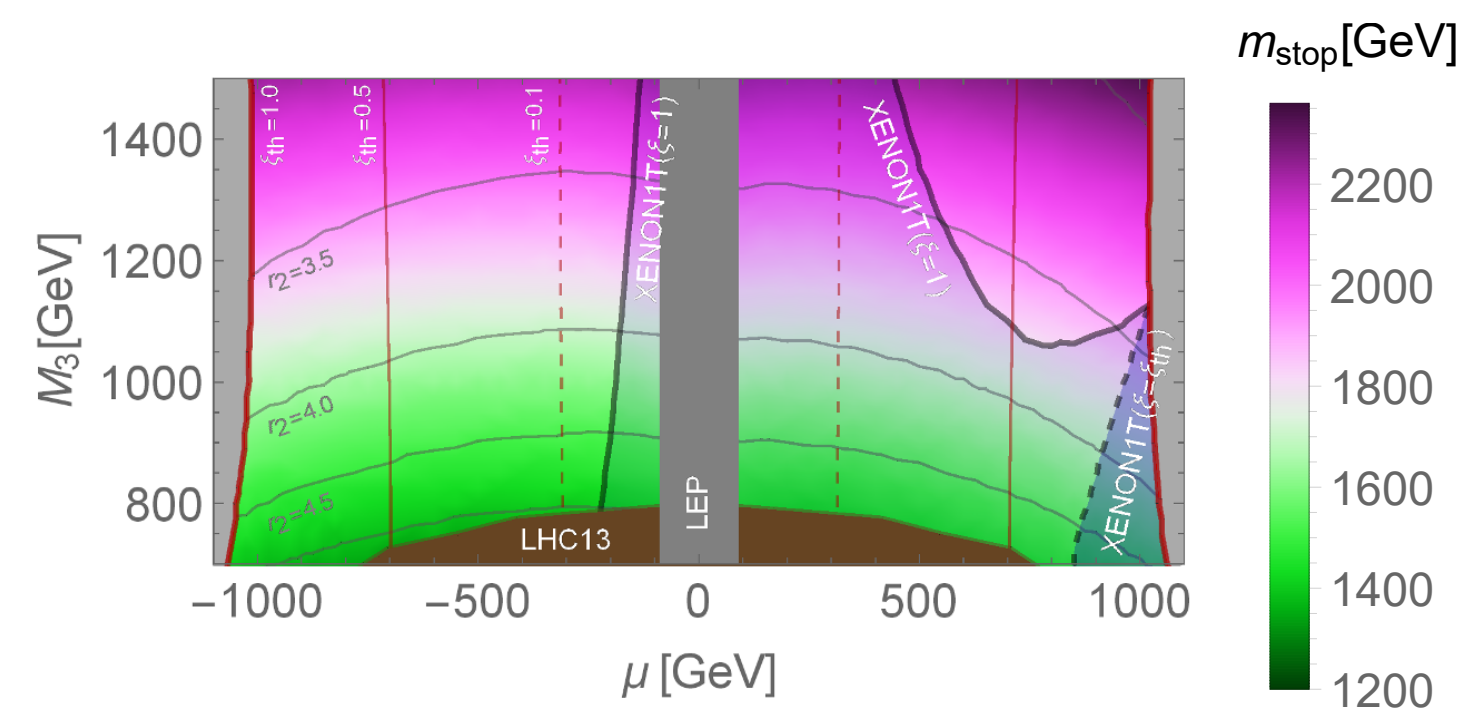

Figure 4. Values of the dark matter observables with $M_{1}=10 \mathrm{TeV}$. Meanings of lines and regions are same as in figure 2. In addition, the dark brown region is excluded by the gluino search at the LHC.

Next, let us discuss the exclusion lines from the spin-independent direct detection. The spin-independent cross section exceeds the current limit given by the LUX experiment [65] in the blue band, assuming that $\xi \equiv \Omega_{\chi} / \Omega_{\mathrm{obs}}=1$ is satisfied on all parameter points. The blue shaded region covered by the solid blue line is the expected limit from the XENON1T experiment in this case. The exclusion limit from the spin-independent cross section becomes stronger as the $\mu$-parameter decreases. The reason is that the experimental limits for the cross section becomes tighter for lighter dark matter masses as long as the dark matter 


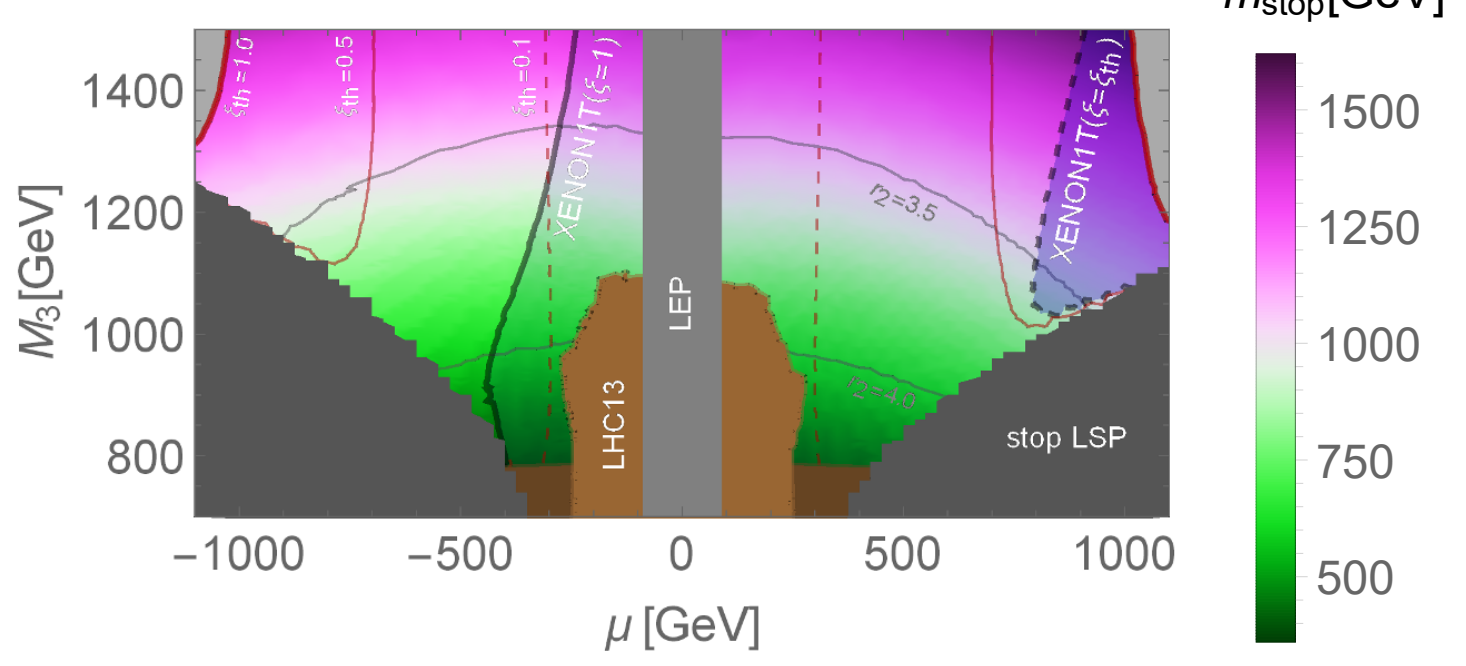

Figure 5. Values of the dark matter observables with $M_{1}=5.0 \mathrm{TeV}$. Meanings of lines and regions are same as in figures $2-4$.

mass is heavier than about $40 \mathrm{GeV}$. The light bino mass region is easier to be excluded due to the large bino-higgsino mixing, especially the well-tempered region has already excluded by the current LUX limit as well known. The spin-independent cross section is significantly large for the positive $\mu$-parameter compared with the case of the negative $\mu$-parameter. This is because the cross section is proportional to $(1+\operatorname{sign}(\mu) \sin 2 \beta)^{2}$ as can be read from eq. (3.9).

Note that the exclusion limits on the $\mu-M_{1}$ plane are severer than the ones derived in ref. [72]. The difference comes from the fact that wino does not decouple completely in the NUGM scenario. The gray lines represent the ratio of wino to gluino mass $r_{2} \equiv M_{2} / M_{3}$ at the unification scale. In order to keep the $\mu$-parameter smaller than $1 \mathrm{TeV}$ motivated by the naturalness problem, the wino mass at the unification scale has to be 3-4 times larger than the gluino mass. The higher wino-to-gluino ratio is required for the lower typical sparticle scale which is defined as the geometric mean of the top squark masses. In this case, $\left(M_{2}, M_{3}\right)$ are about $(4 \mathrm{TeV}, 1.5 \mathrm{TeV})$ at the unification scale and it enhances the spin-independent cross section.

Note that the cross section of the spin-independent direct detection is always larger than $0.25 \times 10^{-10} \mathrm{pb}$ in all figures in this paper. Then, we expect that the future experiments, the XENON1T [70] and the LZ [71], could cover our parameter region as far as $\xi=1$ is satisfied. On the other hand, the current limit from the spin-dependent cross section is fully covered by the spin-independent one.

When we assume that $\Omega_{\chi}$ is only thermally produced, the bound from the direct detection should include the suppression from $\xi_{\text {th }}=\Omega_{\text {th }} / \Omega_{\text {obs. }}$. Taking into account the suppression, the limit would be relaxed as the $\mu$-parameter decreases in the case (A) because of $\xi_{\text {th }}<1$. The region below the white dashed line is excluded by the LUX in this case. The dashed blue line corresponds to the future prospect of the XENON1T experiment 
when the spin-independent direct detection rate is suppressed from $\xi_{\text {th }}$. We see that the exclusion limit is significantly relaxed by this suppression.

Figure 3 shows the allowed region for $\mu$ and $M_{1}$ at $M_{3}=1.0 \mathrm{TeV}$. The different value of $M_{3}$ influences to the direct detection rate and the top squark mass. Top squark becomes the lightest SUSY particle in the dark gray region, and the top squark search at the LHC excludes the brown region. The LHC bounds are projected from the analysis in ref. [9]. The bino mass has to be so large that top squark mass is larger than the higgsino mass.

The lighter gluino mass leads the lighter wino mass and the spin-independent cross section is enhanced by the wino-higgsino mixing. We see that the XENON1T experiment covers the whole region with $\mu>0$ in the case (B) that $\xi=1$ is assumed on all parameter points.

Figures 4 and 5 show the allowed region for $\mu$ and $M_{3}$ where $M_{1}$ is $5.0 \mathrm{TeV}$ and $10.0 \mathrm{TeV}$ at the unification scale, respectively. Other parameters are set to be the same as in figures 2 and 3. The constraint from the gluino search at the LHC is also applied to these figures and it excludes the dark brown region. The gluino mass lower bound is around $800 \mathrm{GeV}$, so that there was no exclusion bounds in figures 2 and 3 . We can see that experimental reaches from direct detections for the gluino mass can be much severer than those from the LHC experiment, assuming $\xi=1$, and $\mu>0$ or $\mu \lesssim 0$.

The wino-higgsino mixing is reduced as gluino becomes heavy. The mixing, however, is not vanishing in our model-dependent analysis. We see that the gaugino-higgsino mixing predicts the spin-independent cross section larger than $2.5 \times 10^{-11}$ pb everywhere in all of the four figures. Thus the parameter region is on the neutrino floor [93] and the region in our analysis would be fully covered by the future planned observations such as the XENON-nT, LZD, PandaX-4T and so on.

\section{Conclusion}

In this paper, we study the dark matter physics in the Non-Universal Gaugino Mass scenario. The NUGM scenario is one of the possible setups of the MSSM to achieve the $125 \mathrm{GeV}$ Higgs boson mass and the $\mu$-parameter below $1 \mathrm{TeV}$, that naturally explain the origin of the EW scale. Since one top squark is relatively light in our scenario, the authors in refs. $[7,8]$ study the current status and the future prospect on the direct search for top squark and gluino at the LHC.

Although the higgsino mass is the most important from the naturalness point of view, higgsino can not be probed by the LHC due to their suitable mass difference $\sim 2 \mathrm{GeV}$. On the other hand, the higgsino mass is critically important for dark matter physics and can be tested by the dark matter observations. The higgsino mass can not be larger than $1 \mathrm{TeV}$ in order not to overclose the universe if we assume that there is no dilution effect after the LSP is frozen out. Furthermore, the degree of tuning the $\mu$-parameter is severer than $\sim 0.4 \%$ if the higgsino is heavier than $1 \mathrm{TeV}$.

Indirect detections for dark matter gives the limits on the higgsino mass independent of other parameters. If the LSP saturates our universe, the AMS-02 experiment have already excluded the higgsino lighter than about $500 \mathrm{GeV}$ even in the most conservative case. This 
limit is tighter than the limits from direct detections especially for negative $\mu$-parameter and very heavy gauginos. On the other hand, there is no limit of the parameter space if the LSP relic density is determined by the thermal production mechanism.

Direct detections for dark matter are powerful tool to probe the neutralino sector of the MSSM. Even the bino and the wino masses are 3-4 TeV, the spin-independent cross section between higgsino and nucleon is in the observational reach. Therefore, the wider parameter space can be covered by the direct detection than the gluino search at the LHC, when the wino-to-gluino mass ratio is fixed to realize the small $\mu$-parameter and the higgsino-like LSP dominates the relic density of dark matter.

If the neutralino density is determined by the standard thermal process, the direct detection is sensitive to the parameter region where the higgsino mass is around $1 \mathrm{TeV}$, while the top squark and the gluino searches at the LHC are generally sensitive to lighter higgsino. Thus the direct detection complement the direct search at the LHC.

The universal gaugino masses are clearly disfavored by the recent dark matter observations. The LSP is either bino or higgsino in this case, but the bino LSP easily overclose the universe. Even if the higgsino LSP is realized in some ways such as considered in refs. [94, 95], light bino and wino are severely constrained by the direct detections. The direct detection constraints push up the gluino mass far above the experimental reach and such a heavy gluino indicates all other sparticles are also hopeless to be discovered except in some special cases. Thus the non-universal gaugino masses with relatively heavy bino and wino masses seems to be more interesting than the universal gaugino masses.

\section{Acknowledgments}

The work of J. K. was supported by Grant-in-Aid for Research Fellow of Japan Society for the Promotion of Science No. 16J04215. The work of Y. O. is supported by Grant-inAid for Scientific research from the Ministry of Education, Science, Sports, and Culture (MEXT), Japan, No. 17H05404.

Open Access. This article is distributed under the terms of the Creative Commons Attribution License (CC-BY 4.0), which permits any use, distribution and reproduction in any medium, provided the original author(s) and source are credited.

\section{References}

[1] S.P. Martin, A supersymmetry primer, in Perspectives on supersymmetry II, G.L. Kane ed., World Scientific, Singapore (1997), hep-ph/9709356 [INSPIRE].

[2] D.J.H. Chung et al., The soft supersymmetry breaking Lagrangian: theory and applications, Phys. Rept. 407 (2005) 1 [hep-ph/0312378] [INSPIRE].

[3] G. Jungman, M. Kamionkowski and K. Griest, Supersymmetric dark matter, Phys. Rept. 267 (1996) 195 [hep-ph/9506380] [INSPIRE].

[4] ATLAS and CMS collaborations, Combined measurement of the Higgs boson mass in pp collisions at $\sqrt{s}=7$ and 8 TeV with the ATLAS and CMS experiments, Phys. Rev. Lett. 114 (2015) 191803 [arXiv:1503.07589] [INSPIRE]. 
[5] H. Abe, T. Kobayashi and Y. Omura, Relaxed fine-tuning in models with non-universal gaugino masses, Phys. Rev. D 76 (2007) 015002 [hep-ph/0703044] [INSPIRE].

[6] H. Abe, J. Kawamura and H. Otsuka, The Higgs boson mass in a natural MSSM with nonuniversal gaugino masses at the GUT scale, PTEP 2013 (2013) $013 \mathrm{~B} 02$ [arXiv: 1208.5328] [INSPIRE].

[7] H. Abe, J. Kawamura and Y. Omura, LHC phenomenology of natural MSSM with non-universal gaugino masses at the unification scale, JHEP 08 (2015) 089 [arXiv: 1505. 03729] [INSPIRE].

[8] J. Kawamura and Y. Omura, Constraints on nonuniversal gaugino mass scenario using the latest LHC data, Phys. Rev. D 93 (2016) 055019 [arXiv:1601.03484] [InSPIRE].

[9] J. Kawamura, Phenomenology of natural supersymmetry with non-universal gaugino masses, Ph.D. thesis, University of Tokyo, Tokyo, Japan (2017).

[10] H. Baer, V. Barger, N. Nagata and M. Savoy, A top-squark hunter's guide, arXiv: 1611.08511 [INSPIRE].

[11] H. Baer, V. Barger, N. Nagata and S. Michael, Phenomenological profile of top squarks from natural supersymmetry at the LHC, Phys. Rev. D 95 (2017) 055012.

[12] H. Baer et al., Gluino reach and mass extraction at the LHC in radiatively-driven natural SUSY, Eur. Phys. J. C 77 (2017) 499 [arXiv:1612.00795] [INSPIRE].

[13] I. Gogoladze, F. Nasir, Q. Shafi and C.S. Un, Nonuniversal gaugino masses and muon $g-2$, Phys. Rev. D 90 (2014) 035008 [arXiv: 1403.2337] [InSPIRE].

[14] K. Choi, A. Falkowski, H.P. Nilles, M. Olechowski and S. Pokorski, Stability of flux compactifications and the pattern of supersymmetry breaking, JHEP 11 (2004) 076 [hep-th/0411066] [INSPIRE].

[15] K. Choi, A. Falkowski, H.P. Nilles and M. Olechowski, Soft supersymmetry breaking in KKLT flux compactification, Nucl. Phys. B 718 (2005) 113 [hep-th/0503216] [INSPIRE].

[16] K. Choi, K.S. Jeong and K.-i. Okumura, Phenomenology of mixed modulus-anomaly mediation in fluxed string compactifications and brane models, JHEP 09 (2005) 039 [hep-ph/0504037] [INSPIRE].

[17] T. Kobayashi, D. Suematsu, K. Yamada and Y. Yamagishi, Nonuniversal soft scalar masses in superstring theories, Phys. Lett. B 348 (1995) 402 [hep-ph/9408322] [INSPIRE].

[18] A. Brignole, L.E. Ibáñez, C. Muñoz and C. Scheich, Some issues in soft SUSY breaking terms from dilaton/moduli sectors, Z. Phys. C 74 (1997) 157 [hep-ph/9508258] [INSPIRE].

[19] A. Brignole, L.E. Ibáñez and C. Muñoz, Soft supersymmetry breaking terms from supergravity and superstring models, Adv. Ser. Direct. High Energy Phys. 18 (1998) 125 [hep-ph/9707209] [INSPIRE].

[20] L. Randall and R. Sundrum, Out of this world supersymmetry breaking, Nucl. Phys. B 557 (1999) 79 [hep-th/9810155] [INSPIRE].

[21] G.F. Giudice, M.A. Luty, H. Murayama and R. Rattazzi, Gaugino mass without singlets, JHEP 12 (1998) 027 [hep-ph/9810442] [INSPIRE].

[22] K. Choi, K.S. Jeong, T. Kobayashi and K.-i. Okumura, Little SUSY hierarchy in mixed modulus-anomaly mediation, Phys. Lett. B 633 (2006) 355 [hep-ph/0508029] [InSPIRE].

[23] K. Choi, K.S. Jeong, T. Kobayashi and K.-i. Okumura, TeV scale mirage mediation and natural little SUSY hierarchy, Phys. Rev. D 75 (2007) 095012 [hep-ph/0612258] [INSPIRE]. 
[24] R. Kitano and Y. Nomura, A solution to the supersymmetric fine-tuning problem within the MSSM, Phys. Lett. B 631 (2005) 58 [hep-ph/0509039] [INSPIRE].

[25] K. Choi, K.Y. Lee, Y. Shimizu, Y.G. Kim and K.-i. Okumura, Neutralino dark matter in mirage mediation, JCAP 12 (2006) 017 [hep-ph/0609132] [INSPIRE].

[26] W.S. Cho, Y.G. Kim, K.Y. Lee, C.B. Park and Y. Shimizu, LHC signature of mirage mediation, JHEP 04 (2007) 054 [hep-ph/0703163] [INSPIRE].

[27] M. Nagai and K. Nakayama, Nonthermal dark matter in mirage mediation, Phys. Rev. D 76 (2007) 123501 [arXiv:0709.3918] [INSPIRE].

[28] S. Nakamura, K.-i. Okumura and M. Yamaguchi, Axionic mirage mediation, Phys. Rev. D 77 (2008) 115027 [arXiv:0803.3725] [INSPIRE].

[29] K. Choi, K.S. Jeong, S. Nakamura, K.-I. Okumura and M. Yamaguchi, Sparticle masses in deflected mirage mediation, JHEP 04 (2009) 107 [arXiv:0901.0052] [INSPIRE].

[30] M. Holmes and B.D. Nelson, Dark matter prospects in deflected mirage mediation, JCAP 07 (2009) 019 [arXiv:0905.0674] [InSPIRE].

[31] B. Altunkaynak, B.D. Nelson, L.L. Everett, I.-W. Kim and Y. Rao, Phenomenological implications of deflected mirage mediation: comparison with mirage mediation, JHEP 05 (2010) 054 [arXiv: 1001.5261] [INSPIRE].

[32] L.L. Everett, I.-W. Kim, P. Ouyang and K.M. Zurek, Moduli stabilization and supersymmetry breaking in deflected mirage mediation, JHEP 08 (2008) 102 [arXiv:0806.2330] [INSPIRE].

[33] L.L. Everett, I.-W. Kim, P. Ouyang and K.M. Zurek, Deflected mirage mediation: a framework for generalized supersymmetry breaking, Phys. Rev. Lett. 101 (2008) 101803 [arXiv: 0804.0592] [INSPIRE].

[34] M. Asano and T. Higaki, Natural supersymmetric spectrum in mirage mediation, Phys. Rev. D 86 (2012) 035020 [arXiv:1204.0508] [INSPIRE].

[35] T. Kobayashi, H. Makino, K.-i. Okumura, T. Shimomura and T. Takahashi, TeV scale mirage mediation in NMSSM, JHEP 01 (2013) 081 [arXiv:1204.3561] [INSPIRE].

[36] H. Abe and J. Kawamura, The $126 \mathrm{GeV}$ Higgs boson mass and naturalness in (deflected) mirage mediation, JHEP 07 (2014) 077 [arXiv: 1405.0779] [INSPIRE].

[37] K. Hagimoto, T. Kobayashi, H. Makino, K.-i. Okumura and T. Shimomura, Phenomenology of NMSSM in TeV scale mirage mediation, JHEP 02 (2016) 089 [arXiv:1509.05327] [INSPIRE].

[38] L.L. Everett, T. Garon, B.L. Kaufman and B.D. Nelson, Mirage models confront the LHC: III. Deflected mirage mediation, Phys. Rev. D 93 (2016) 055031 [arXiv:1510.05692] [INSPIRE].

[39] V. Barger, L.L. Everett and T.S. Garon, Electroweak naturalness and deflected mirage mediation, Phys. Rev. D 93 (2016) 075024 [arXiv:1512.05011] [INSPIRE].

[40] H. Baer, V. Barger, H. Serce and X. Tata, Natural generalized mirage mediation, Phys. Rev. D 94 (2016) 115017 [arXiv: 1610.06205] [INSPIRE].

[41] J.E. Younkin and S.P. Martin, Non-universal gaugino masses, the supersymmetric little hierarchy problem and dark matter, Phys. Rev. D 85 (2012) 055028 [arXiv:1201.2989] [INSPIRE].

[42] R. Blumenhagen, B. Körs, D. Lüst and S. Stieberger, Four-dimensional string compactifications with D-branes, orientifolds and fluxes, Phys. Rept. 445 (2007) 1 [hep-th/0610327] [INSPIRE]. 
[43] R. Barbieri and G.F. Giudice, Upper bounds on supersymmetric particle masses, Nucl. Phys. B 306 (1988) 63 [INSPIRE].

[44] J. Kawamura and Y. Omura, in preparation.

[45] M. Ibe, T. Moroi and T.T. Yanagida, Possible signals of Wino LSP at the Large Hadron Collider, Phys. Lett. B 644 (2007) 355 [hep-ph/0610277] [INSPIRE].

[46] R. Mahbubani, P. Schwaller and J. Zurita, Closing the window for compressed dark sectors with disappearing charged tracks, JHEP 06 (2017) 119 [arXiv:1703.05327] [INSPIRE].

[47] H. Fukuda, N. Nagata, H. Otono and S. Shirai, Higgsino dark matter or not: role of disappearing track searches at the LHC and future colliders, arXiv:1703.09675 [INSPIRE].

[48] ATLAS collaboration, Search for bottom squark pair production with the ATLAS detector in proton-proton collisions at $\sqrt{s}=13 \mathrm{TeV}$, ATLAS-CONF-2015-066 (2015).

[49] ATLAS collaboration, Search for the supersymmetric partner of the top quark in the jets+emiss final state at $\sqrt{s}=13 \mathrm{TeV}$, ATLAS-CONF-2016-077 (2016).

[50] ATLAS collaboration, Search for pair production of gluinos decaying via top or bottom squarks in events with b-jets and large missing transverse momentum in pp collisions at $\sqrt{s}=13 \mathrm{TeV}$ with the ATLAS detector, ATLAS-CONF-2016-052 (2016).

[51] ATLAS collaboration, Further searches for squarks and gluinos in final states with jets and missing transverse momentum at $\sqrt{s}=13 \mathrm{TeV}$ with the ATLAS detector, ATLAS-CONF-2016-078 (2016).

[52] M. Cirelli, N. Fornengo and A. Strumia, Minimal dark matter, Nucl. Phys. B 753 (2006) 178 [hep-ph/0512090] [INSPIRE].

[53] M. Cirelli, A. Strumia and M. Tamburini, Cosmology and astrophysics of minimal dark matter, Nucl. Phys. B 787 (2007) 152 [arXiv:0706.4071] [inSPIRE].

[54] N. Arkani-Hamed, A. Delgado and G.F. Giudice, The well-tempered neutralino, Nucl. Phys. B 741 (2006) 108 [hep-ph/0601041] [INSPIRE].

[55] K. Hamaguchi and K. Ishikawa, Prospects for Higgs- and Z-resonant neutralino dark matter, Phys. Rev. D 93 (2016) 055009 [arXiv:1510.05378] [INSPIRE].

[56] Planck collaboration, P.A.R. Ade et al., Planck 2015 results. XIII. Cosmological parameters, Astron. Astrophys. 594 (2016) A13 [arXiv:1502.01589] [INSPIRE].

[57] K. Kohri, M. Yamaguchi and J. Yokoyama, Neutralino dark matter from heavy gravitino decay, Phys. Rev. D 72 (2005) 083510 [hep-ph/0502211] [INSPIRE].

[58] H. Baer, A. Lessa and W. Sreethawong, Coupled Boltzmann calculation of mixed axion/neutralino cold dark matter production in the early universe, JCAP 01 (2012) 036 [arXiv:1110.2491] [INSPIRE].

[59] H. Baer, K.-Y. Choi, J.E. Kim and L. Roszkowski, Dark matter production in the early Universe: beyond the thermal WIMP paradigm, Phys. Rept. 555 (2015) 1 [arXiv:1407.0017] [INSPIRE].

[60] R. Allahverdi, M. Cicoli, B. Dutta and K. Sinha, Nonthermal dark matter in string compactifications, Phys. Rev. D 88 (2013) 095015 [arXiv:1307.5086] [INSPIRE].

[61] M. Garny, A. Ibarra, M. Pato and S. Vogl, On the spin-dependent sensitivity of XENON100, Phys. Rev. D 87 (2013) 056002 [arXiv:1211.4573] [INSPIRE].

[62] XENON100 collaboration, E. Aprile et al., Dark matter results from 225 live days of XENON100 data, Phys. Rev. Lett. 109 (2012) 181301 [arXiv:1207.5988] [INSPIRE]. 
[63] XENON100 collaboration, E. Aprile et al., Limits on spin-dependent WIMP-nucleon cross sections from 225 live days of XENON100 data, Phys. Rev. Lett. 111 (2013) 021301 [arXiv:1301.6620] [INSPIRE].

[64] LUX collaboration, D.S. Akerib et al., Improved limits on scattering of weakly interacting massive particles from reanalysis of 2013 LUX data, Phys. Rev. Lett. 116 (2016) 161301 [arXiv: 1512.03506] [INSPIRE].

[65] LUX collaboration, D.S. Akerib et al., Results from a search for dark matter in the complete LUX exposure, Phys. Rev. Lett. 118 (2017) 021303 [arXiv: 1608. 07648] [INSPIRE].

[66] PandaX-II collaboration, A. Tan et al., Dark matter results from first 98.7 days of data from the PandaX-II experiment, Phys. Rev. Lett. 117 (2016) 121303 [arXiv:1607.07400] [INSPIRE].

[67] PandaX-II collaboration, C. Fu et al., Spin-dependent weakly-interacting-massiveparticle-nucleon cross section limits from first data of PandaX-II experiment, Phys. Rev. Lett. 118 (2017) 071301 [arXiv: 1611.06553] [INSPIRE].

[68] PICO collaboration, C. Amole et al., Dark matter search results from the PICO-60 $\mathrm{CF}_{3} I$ bubble chamber, Phys. Rev. D 93 (2016) 052014 [arXiv:1510.07754] [INSPIRE].

[69] PICO collaboration, C. Amole et al., Improved dark matter search results from PICO-2L Run 2, Phys. Rev. D 93 (2016) 061101 [arXiv:1601.03729] [INSPIRE].

[70] XENON collaboration, E. Aprile et al., Physics reach of the XENON1T dark matter experiment, JCAP 04 (2016) 027 [arXiv: 1512.07501] [INSPIRE].

[71] LZ collaboration, D.S. Akerib et al., LUX-ZEPLIN (LZ) conceptual design report, arXiv: 1509.02910 [INSPIRE].

[72] C. Cheung, L.J. Hall, D. Pinner and J.T. Ruderman, Prospects and blind spots for neutralino dark matter, JHEP 05 (2013) 100 [arXiv:1211.4873] [INSPIRE].

[73] G. Bélanger, F. Boudjema, A. Pukhov and A. Semenov, MicrOMEGAs_3: a program for calculating dark matter observables, Comput. Phys. Commun. 185 (2014) 960 [arXiv: 1305.0237] [INSPIRE].

[74] G. Bélanger, F. Boudjema, A. Pukhov and A. Semenov, MicrOMEGAs: version 1.3, Comput. Phys. Commun. 174 (2006) 577 [hep-ph/0405253] [INSPIRE].

[75] G. Bélanger, F. Boudjema, A. Pukhov and A. Semenov, MicrOMEGAs: a program for calculating the relic density in the MSSM, Comput. Phys. Commun. 149 (2002) 103 [hep-ph/0112278] [INSPIRE].

[76] G. Bélanger, F. Boudjema, A. Pukhov and A. Semenov, MicrOMEGAs4.1: two dark matter candidates, Comput. Phys. Commun. 192 (2015) 322 [arXiv:1407.6129] [INSPIRE].

[77] G. Bélanger, F. Boudjema, A. Pukhov and A. Semenov, Dark matter direct detection rate in a generic model with MicrOMEGAs 2.2, Comput. Phys. Commun. 180 (2009) 747 [arXiv: 0803.2360] [INSPIRE].

[78] M. Drees and M. Nojiri, Neutralino-nucleon scattering revisited, Phys. Rev. D 48 (1993) 3483 [hep-ph/9307208] [INSPIRE].

[79] J. Hisano, K. Ishiwata, N. Nagata and T. Takesako, Direct detection of electroweak-interacting dark matter, JHEP 07 (2011) 005 [arXiv: 1104.0228] [INSPIRE].

[80] ICECube collaboration, M.G. Aartsen et al., Search for annihilating dark matter in the Sun with 3 years of IceCube data, Eur. Phys. J. C 77 (2017) 146 [arXiv:1612.05949] [inSPIRE]. 
[81] Fermi-LAT collaboration, M. Ackermann et al., Searching for dark matter annihilation from Milky Way dwarf spheroidal galaxies with six years of Fermi Large Area Telescope data, Phys. Rev. Lett. 115 (2015) 231301 [arXiv:1503.02641] [INSPIRE].

[82] AMS collaboration, M. Aguilar et al., Antiproton flux, antiproton-to-proton flux ratio, and properties of elementary particle fluxes in primary cosmic rays measured with the alpha magnetic spectrometer on the international space station, Phys. Rev. Lett. 117 (2016) 091103.

[83] A. Cuoco, M. Krämer and M. Korsmeier, Novel dark matter constraints from antiprotons in light of AMS-02, Phys. Rev. Lett. 118 (2017) 191102 [arXiv:1610.03071] [INSPIRE].

[84] M.-Y. Cui, Q. Yuan, Y.-L.S. Tsai and Y.-Z. Fan, Possible dark matter annihilation signal in the AMS-02 antiproton data, Phys. Rev. Lett. 118 (2017) 191101 [arXiv:1610.03840] [INSPIRE].

[85] G.A. Gómez-Vargas et al., Constraints on WIMP annihilation for contracted dark matterin the inner Galaxy with the Fermi-LAT, JCAP 10 (2013) 029 [arXiv:1308.3515] [INSPIRE].

[86] CTA collaboration, J. Carr et al., Prospects for Indirect Dark Matter Searches with the Cherenkov Telescope Array (CTA), PoS(ICRC2015)1203 [arXiv: 1508.06128] [INSPIRE].

[87] L. Aparicio, M. Cicoli, B. Dutta, F. Muia and F. Quevedo, Light Higgsino dark matter from non-thermal cosmology, JHEP 11 (2016) 038 [arXiv:1607.00004] [INSPIRE].

[88] B.C. Allanach, SOFTSUSY: a program for calculating supersymmetric spectra, Comput. Phys. Commun. 143 (2002) 305 [hep-ph/0104145] [INSPIRE].

[89] A. Djouadi, M.M. Muhlleitner and M. Spira, Decays of supersymmetric particles: the program SUSY-HIT (SUspect-SdecaY-HDECAY-InTerface), Acta Phys. Polon. B 38 (2007) 635 [hep-ph/0609292] [INSPIRE].

[90] M. Muhlleitner, A. Djouadi and Y. Mambrini, SDECAY: a Fortran code for the decays of the supersymmetric particles in the MSSM, Comput. Phys. Commun. 168 (2005) 46 [hep-ph/0311167] [INSPIRE].

[91] A. Djouadi, J. Kalinowski and M. Spira, HDECAY: a program for Higgs boson decays in the standard model and its supersymmetric extension, Comput. Phys. Commun. 108 (1998) 56 [hep-ph/9704448] [INSPIRE].

[92] K. Jakobs, MSSM SUSY searches at LEP-2, hep-ex/0107084 [INSPIRE].

[93] J. Billard, L. Strigari and E. Figueroa-Feliciano, Implication of neutrino backgrounds on the reach of next generation dark matter direct detection experiments, Phys. Rev. D 89 (2014) 023524 [arXiv: 1307.5458] [INSPIRE].

[94] J.L. Feng and D. Sanford, A natural 125 GeV Higgs boson in the MSSM from focus point supersymmetry with A-terms, Phys. Rev. D 86 (2012) 055015 [arXiv:1205.2372] [INSPIRE].

[95] H. Baer, V. Barger, P. Huang, A. Mustafayev and X. Tata, Radiative natural SUSY with a 125 GeV Higgs boson, Phys. Rev. Lett. 109 (2012) 161802 [arXiv:1207.3343] [INSPIRE]. 